\title{
Methionine Restriction and Modification of Epithelial Tight Junction Barrier Function and Permeability
}

\author{
Xuexuan Wang ${ }^{1}$ and James M. Mullin ${ }^{1,2, *}$ \\ ${ }^{1}$ Lankenau Institute for Medical Research, 100 Lancaster Ave, Wynnewood, PA 19096 USA \\ ${ }^{2}$ Division of Gastroenterology, Lankenau Medical Center, 100 Lancaster Avenue, Wynnewood, PA 19096 USA
}

\begin{abstract}
It is well known that caloric restriction leads to an increased longevity by forestalling age-related diseases. Dietary restriction of methionine also renders similar benefits. In this review, we report studies on the effect of methionine restriction on epithelial barrier function in a renal epithelial LLC-PK 1 cell, a rodent gastrointestinal model and a clinical trial using Crohn's patients. In LLC-PK 1 cell culture, a reduction of culture medium methionine by $80 \%$ resulted in altered tight junctional claudin composition with improved epithelial barrier function as exemplified by increased transepithelial electrical resistance and decreased paracellular leak to ${ }^{14} \mathrm{C}-D$-mannitol. In addition to small but significant reductions in plasma and intracellular colonocyte methionine levels, dietary methionine restriction tightens intestinal epithelium and reduces mannitol permeability in rat colonic, but not ileal tissue. Crohn's patients on methionine-restricted diet reported improved symptoms as demonstrated by a reduced CDAI index, but this diet was not able to significantly reduce the plasma methionine level in patients recruited. An increased urinary lactulose/mannitol ratio was also the result of the regimen in Crohn's patients, suggesting an increased, rather than decreased ileal leakage. Overall, our results showed that reduction in dietary intake of methionine is promising in improving at least some epithelial barriers and this may be through altering tight junctional protein compositions. Methionine restriction might result in contrasting effects depending on the tissue type and possible disease conditions, thus wider range of studies are required.
\end{abstract}

Keywords: Methionine, tight junction, $\mathrm{LLC}^{\mathrm{PK}}{ }_{1}$, Crohn's disease, claudin, occludin, amino acid, colon, ileum, kidney.

\section{METHIONINE RESTRICTION IN AGING AND CAN- CER STUDIES}

Clive McCay and colleagues reported in 1935 that the life span of rats increased by decreasing their food intake [1], making it more than 70 years since caloric restriction (CR) or dietary restriction was found to consistently increase longevity in a range of animal models [2-4]. Although its modes of action are still under investigation [2], a similar effect can be achieved in rodents through a diet low in the essential amino acid, methionine (Met) (with an elimination of related sulfur-containing amino acids, cysteine and cystine) [6-8]. In a recent study using fruit fly Drosophila melanogaster, the addition of Met to a CR diet reduced life span and this reduction in life span could be avoided by simply removing Met from the diet [3]. Both nutritional interventions, CR and methionine restriction (MR), do not only simply extend life span, but also forestall the onset of a variety of age-related diseases, such as cancers, obesity, diabetes, hypercholesterolemia and more [3, 4, 6, 9-11]. Since the etiology of many types of diseases involves collapse of epithelial and/or endothelial barriers [4], it is reasonable to speculate that either CR or MR, at least in part, exert their benefits through strengthening barrier functions. In this review, we are going to focus on MR and its effects on renal and intestinal barriers.

\footnotetext{
*Address correspondence to this author at the Lankenau Institute for Medical Research, 100 Lancaster Ave Rm 229, Wynnewood, PA 19096 USA; Tel: 484-476-2903; Fax: 484-476-2205; E-mail: mullinj@mlhs.org
}

In addition to its role in protein synthesis, Met is critical in generating $S$-adenosylmethionine (SAM), the major methyl donor for a variety of biologically important reactions. After donating its methyl group, SAM is converted to $S$-adenosylhomocysteine which is in turn hydrolyzed to form homocysteine. Homocysteine can either be re-methylated to Met, or enter the trans-sulfuration pathway to form cysteine and then produce an important intracellular anti-oxidant, glutathione. Unlike normal cells, the maintenance of cancer cells is much more reliant on Met, a pivotal nutrient for tumor growth, as transformed cells are relatively incapable of transforming compensating homocysteine into Met [5]. This observation has lead to several studies focusing on the synergistic effect of MR and traditional cancer therapies through either dietary restriction of Met or by administration of methioninase (L-methionine- $\alpha$-deamino- $\gamma$-lyase) [14, 15]. A number of studies on cell cultures using Met-restricted media demonstrated that MR suppresses tumor cell growth, while little harmful effect was observed on normal cells [6-8]. Similarly, a tumor inhibition effect was also observed in animal models in vivo. In a rat colon cancer model, for example, MR has been shown to inhibit the formation of preneoplastic aberrant crypt foci after the administration of the carcinogen azoxymethane [9]. Data from a phase I clinical trial conducted on 8 patients with metastatic solid tumors by Epner and colleagues indicated for the first time that diet with a reduced Met content is safe and feasible to treat patients with cancer, despite a conclusive lack of sufficient anti-tumor activity $[10,11]$. A Met-free diet was used in con- 
junction with other anti-cancer agents in another two Phase I clinical trials $[12,13]$. Both regimens were found to be relatively feasible and well tolerated, one of which resulted in partial response to the therapy and disease stabilization among 4 evaluated patients [13]. Despite the small patient number, these trials demonstrated for the first time in humans that MR has promise for enhancing the efficacy of chemotherapy.

In addition to its synergistic effect in chemotherapy of original tumors, MR has also demonstrated its ability in decreasing the motility and invasiveness of existing cancer cells $[6,14,15]$. Studies using murine or human melanoma cells showed that MR suppresses attachment and motility of melanoma cells by the inhibition of specific integrin expression, in turn through integrin-mediated focal adhesion kinase (FAK) phosphorylation [6]. In prostate cancer cells, MR not only induced apoptosis, but also reduced the invasiveness of two different prostate cell lines [16]. This effect seemed to be via a FAK/extracellular-regulated kinase (ERK)-dependent pathway [16] and Raf/Akt survival pathway [17]. MR also modulates g-protein activity and the relative abundance and phosphorylation of the motility proteins cofilin and profilin [14]. Since the effect of Akt on cell survival requires glucose metabolism, the latest research from the same group revealed that MR-induced cell death depends on alterations of glucose metabolism [18].

The underlying mechanism by which MR exerts its effects on either aging or cancers still needs to be resolved. However some specific metabolic sensors may be involved in response to the concentration changes of circulating and/or tissue levels of Met and its various metabolites. Many of those sensors are extremely well conserved in evolution and often act autonomously within the cell. They possibly include the target of rapamycin (TOR) [19], AMPactivated protein kinase (AMPK) [20], and the "anti-aging" sirtuin proteins (SIT2 or SIRT1 in mammals specifically) $[21,22]$. They detect specific metabolic changes in amino acids, AMP and $\mathrm{NAD}^{+}$, respectively. These sensors are sometimes tightly intertwined [23], making pathway identification of MR or CR mechanisms more intricate. Instead of regulating a large number of downstream sensory and signaling genes individually to achieve longevity, research has shown that SAM could modulate those genes in general by binding to riboswitches and/or affecting DNA methylation/ polyamine synthesis [24].

\section{THE EFFECT OF METHIONINE RESTRICTION ON EPITHELIAL TIGHT JUNCTIONS}

Despite the lack of exact mechanism, the positive effects of MR in mammalian biology and physiology are apparent. We propose that such benefits may in part involve the improvement of the overall epithelial barrier effectiveness, by making the barriers less affected by environmental and microbial stress and challenges. To this end, MR effects on the epithelial tight junction (TJ) apparatus are a pivotal component that deserves attention.

Depending on the specific organ, epithelial tissue of any kind either separates the external environment from the bloodstream (such as skin, cornea and nasal mucosae), or separates luminal internal fluids from the bloodstream, such as in the gastrointestinal (GI) and urinary tracts. These barriers are not impermeable however. For example, xenobiotics including bacterial toxins can gain entry into the inner (vascular) compartment through either transcellular or paracellular routes. Tight junctions (or zonula occludens) are positioned at the apical end of the epithelial lateral intercellular space, serving as a selectively permeable barrier governing the degree of paracellular leakiness [25, 26]. Disease often results in leaky epithelial barriers, allowing abnormally increased paracellular permeability of substances [4]. This makes discovering methods that can improve barrier function very clinically desirable. The following sections summarize and discuss our studies on MR in modulating TJs and epithelial barriers in cell culture, animal models and a small clinical trial.

\section{MR EFFECTS ON THE LLC-PK 1 RENAL EPITHELIUM}

Attempting to investigate the effect of MR diet on epithelial TJ barriers, we began by mimicking the MR diet used in longevity studies in vivo for use with the $\mathrm{LLC}^{-\mathrm{PK}_{1}}$ renal epithelial cell line in vitro. A Met-free, cysteine-free and cystine-free alpha-modified "base" MEM medium was used with $10 \%$ dialyzed fetal bovine serum (to eliminate any sulfur-containing amino acids contributed from the serum) [27]. A final formulation of $0.285 \mathrm{mM}$ cysteine $(50 \%), 0 \mathrm{mM}$ cystine, and $0.01 \mathrm{mM}$ Met (10\% of normal alpha-MEM levels) was able to allow normal cell division, polarization and differentiation. This was exemplified by the presence of lumen negative, $1 \mathrm{mV}$ potential differences, apical microvilli and $\mathrm{Na}^{+}$-dependent sugar transport, hallmark features of LLC$\mathrm{PK}_{1}$ epithelium [28]. Vectorial salt and fluid transport was evident in post-confluent LLC-PK 1 epithelium cultured with the modified MR medium (see the domes in Fig. 1), with minor differences in morphology compared to cells in control medium.

Continuous TJs were confirmed by immunoflourescent staining of ZO-1, an important TJ-associated protein, in both media (Fig. 2). The cell number per unit was also counted using this method and equal cell number per unit area was calculated under both conditions. This suggests an equivalent liner junctional density in both Met-restricted and control media. Based on this, the significantly higher transepithelial electrical resistance $\left(\mathrm{R}_{\mathrm{t}}\right)$ that was observed in confluent LLC$\mathrm{PK}_{1}$ epithelium in MR medium (Fig. 3A) is not due to a junctional density difference. Furthermore, the considerably tighter epithelium grown in MR medium was accompanied by an unchanged short-circuit current $\left(\mathrm{I}_{\mathrm{scc}}\right)$. This indicates that the transepithelial $\mathrm{Na}^{+}$transport activity at both apical and basolateral side of the cell sheet was not altered by a reduced Met level in the cell culture medium (Fig. 3B). Apical-to-basolateral flux of ${ }^{14} \mathrm{C}-D$-mannitol, a paracellular transport marker, was also measured to generate further support for an MR-induced change in TJ permeability (Fig. 3C). A nearly $75 \%$ reduction was observed in the transepithelial mannitol diffusion rate in the MR condition implying an appreciable decrease in the leakiness of the LLC-PK 1 TJs to non-electrolytes.

To further confirm that the increased $\mathrm{R}_{\mathrm{t}}$ and decreased mannitol permeability seen in Met restricted $\mathrm{LLC}_{\mathrm{P}} \mathrm{PK}_{1}$ cells is due to modified TJs, a few TJ protein abundance assays 


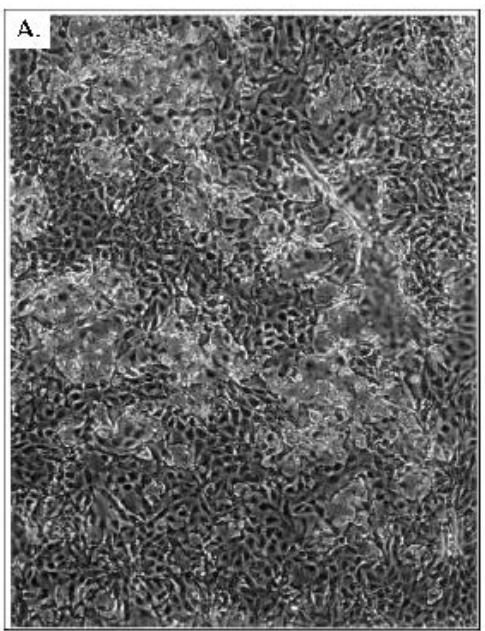

Control

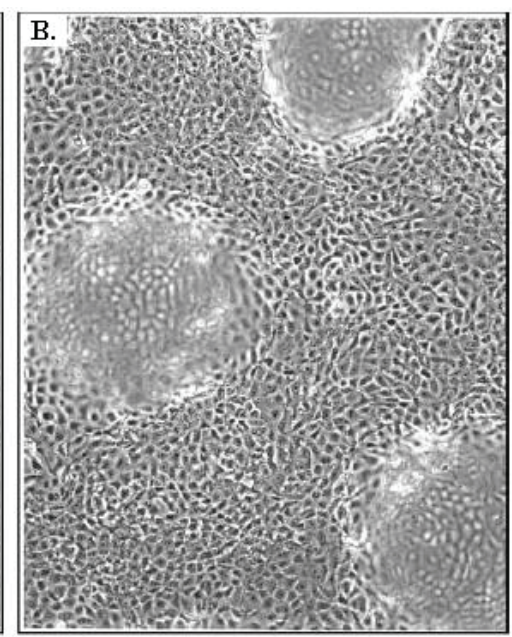

MR

Fig. (1). Phase contrast micrograph of the one week postconfluent cultures of control vs. MR conditions. Cells were seeded into $75 \mathrm{~cm}^{2}$ tissue culture flasks and allowed to reach confluence (one week). Flasks were than re-fed in either control (100\% cycsteine/ $100 \%$ cystine $/ 100 \%$ Met) or Met-restricted (50\% cycsteine $/ 0 \%$ cystine $/ 10 \%$ Met) media on the day of confluence, again 4 days later and again at one week postconfluence. Results are typical and show the three-dimensional, fluid-filled domes characteristic of confluent LLC$\mathrm{PK}_{1}$ cultures, with the domes being larger in MR medium (B). The control condition (A) resembles a typical one week postconfluent LLC$\mathrm{PK}_{1}$ cell sheet with numerous but very small domes; the MR condition appears more like a typical freshly confluent LLC-PK 1 cell sheet. Since in LLC-PK 1 , doming is indicative of a healthy, polarized cell sheet that is actively and transepithelially transporting ions. This photograph suggests that MR medium did not have negative impact on the basic functions of LLC-PK 1 cells as transporting epithelia.

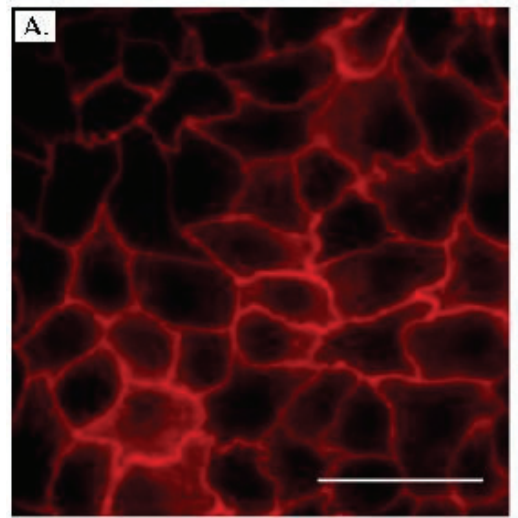

Control

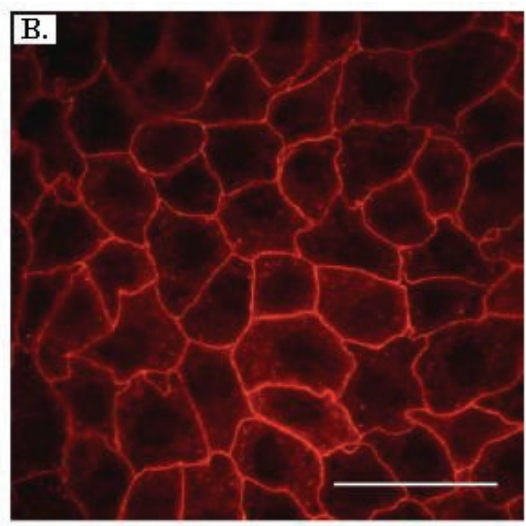

MR

Fig. (2). Immunoflourescence micrographs of LLC-PK 1 cell sheets fed with control (A) vs. MR (B) medium. Cells were seeded at confluent density on permeable $(0.4 \mu \mathrm{m}$ pore size) Millipore PCF filter ring assemblies and after 24 hours kept on control or MR media for one week. Filters were then excised from the rings and stained with Cy3-labeled $2^{\circ}$ antibody against the $1^{\circ}$ antibody for the junction-specific protein, ZO-1. Scale bar $=20 \mu \mathrm{m}$.

were conducted. Western immunoblots on transmembrane TJ proteins occludin and claudins were chosen because they are key components of the actual physiological barrier function of the TJ $[29,30]$. As shown in Fig. (4), MR did not significantly change the abundance of total occludin or claudin- 1 or -2 , but it did induce noticeable decreases in the abundance of claudin-3 and -7 in LLC-PK 1 epithelium, and significant increases in the abundance of claudin-4 and -5 . Although 1) the changes in remaining claudin proteins and any phosphorylation status of these proteins are still to be examined, and 2) so far there is no definitive link between particular TJ protein changes and permeability changes, a clear pattern could still be drawn that MR is changing the structural components of the TJ barrier and the permeability of the barrier. These changes are one of extremely few inter- ventions that are known to improve, rather than compromise, TJ barrier function [31].

\section{MR EFFECTS ON RAT SMALL INTESTINE AND COLON}

In order to extend our previous renal epithelial cell culture studies on restricted Met to a more medically relevant and a less reductionist system, we chose to follow the LLC$\mathrm{PK}_{1}$ study with a rat model to investigate whether MR also enhances epithelial barrier properties in gastrointestinal tissue.

Similar to the diet used by Richie et al., (2004) in their longevity studies, a diet with protein (casein) content 

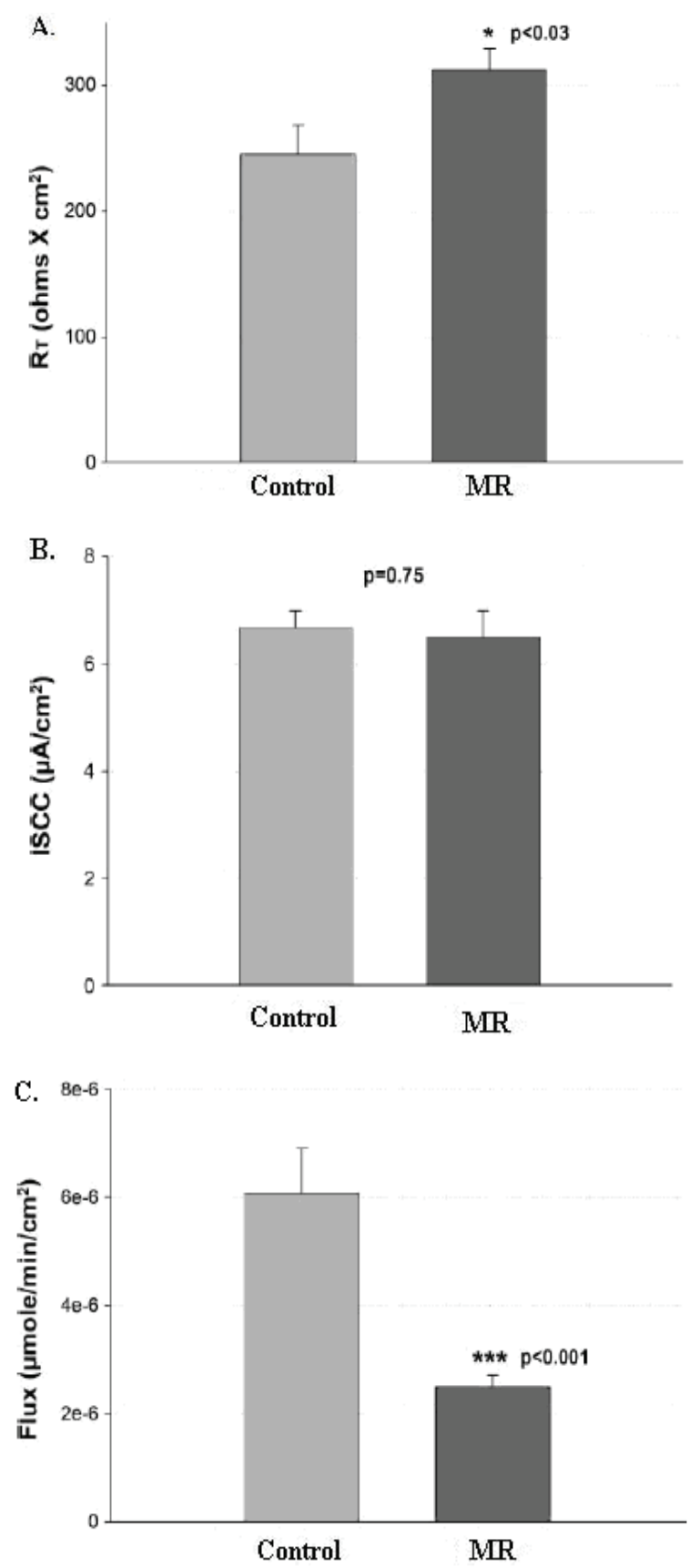

Fig. (3). MR medium-treated $L L C-P K_{1}$ cell sheets exhibit a significant increase in $R_{t}(A)$, a comparable $I_{\text {scc }}(B)$, and a significant decrease in transepithelial ${ }^{14} \mathrm{C}$ - $D$-mannitol flux $(C)$, as compared to controls. Cells were seeded at confluent density onto permeable $(0.4 \mu \mathrm{m}$ pore size) Millipore PCF filter ring assemblies and after 24 hours kept on control or MR media for one week before potential difference $(\mathrm{PD})$ and $\mathrm{I}_{\mathrm{scc}}$ readings were taken using $\mathrm{Ag} / \mathrm{AgCl}$ electrodes in series with $\mathrm{NaCl}$ agar bridges and a modified Ussing chamber set-up, respectively. Ohm's law was used to calculate $\mathrm{R}_{\mathrm{t}}$. The radiotracer probe, ${ }^{14} \mathrm{C}$-mannitol, was put in the dish media basolaterally and allowed to incubate for 3 hours, during which time samples were taken from the ring media apically. Flux was calculated using linear regression of cpm vs. time. Data shown represent the mean \pm standard error of the mean (SEM), $n=9$ cell sheets. $*$ and $* * *$ denote $\mathrm{p}<0.05$ and $\mathrm{p}<0.001$ significances, respectively, as tested by Student's $t$-test, assuming equal variances. replaced by an essential amino acid mixture was used [32]. The MR diet contained $20 \%$ of the Met that is present in the control diet. For the same reason as in the cell culture study, other (non-essential) sulfur-containing amino acids (cysteine and cystine) were removed from MR diet. Rats (starting weight of $\sim 400 \mathrm{~g}$ and $7-8$ weeks old) in both groups had free access to food and water. The reduction of the overall nitrogen content of the diet as result of MR was compensated by a minor increase in glutamate level [33].

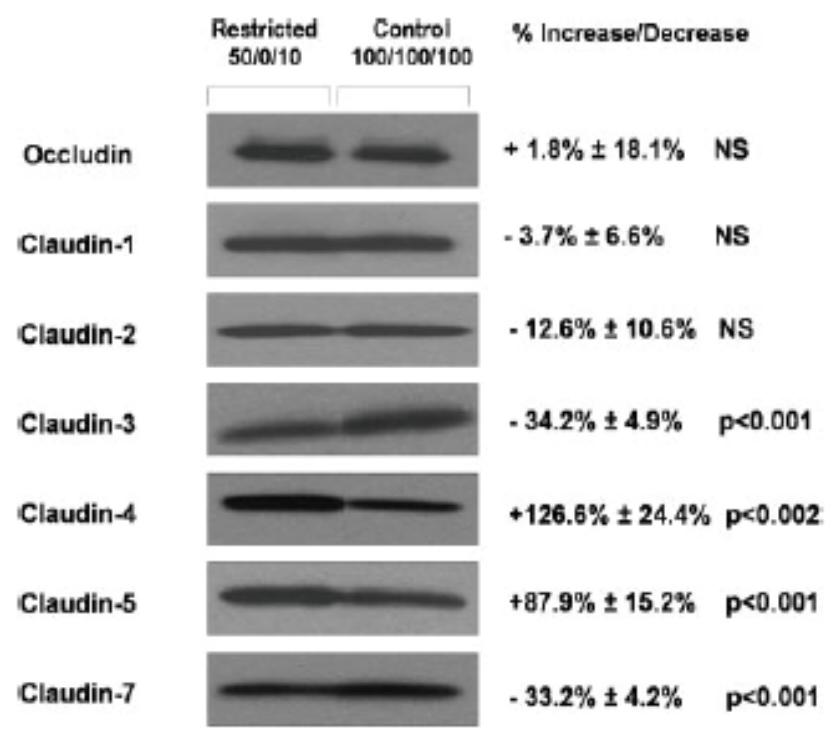

Fig. (4). Western immunoblots of integral TJ proteins in control and MR-treated LLC-PK 1 cell sheets. Cell sheets in the same condition as described in Fig.1 were scraped and processed for Western blots. Results shown are from the membrane fraction (Triton-soluble) and representative of $n=4$ runs using four different cell passages. The percentage changes represent the mean \pm SEM and were calculated by densitometry of the appropriate bands of the four separate PAGE and Western immunoblots. Significance was determined using Student's $t$-test, assuming equal variances.

Based on the observed appearance and activity level, rats in both groups appeared healthy throughout the experimental period (from 3-4 days to up to 9 weeks) [34]. While rats on control chow gained weight consistently, those on MR diet lost about $10 \%$ of their initial weight for the first 2-3 weeks before subsequently beginning to gain weight. However the weight was maintained at approximately $93 \%$ of the starting weight by the end of the 9 -week study (Fig. 5). Although the initial weight dip has never been reported by others, previous studies did indicate that chronic dietary MR reduces the deposition of adipose tissue [35, 36]. A recent study by Hasek et al., (2010) also showed a significant decrease in body weight and adiposity of MR rats relative to the control group after the initial 2 weeks [37]. The authors speculated that this is possibly due to a metabolic inefficiency with higher maintenance requirements induced by MR diet. In the same report, researchers also documented that when MR diet was applied to rats after physical maturity (6 month of age), they did not increase body weight during the 26-week long study, maintaining $96 \%$ of their initial weight.

With the exception of glucose and BUN (blood urea nitrogen) levels, all plasma electrolytes and non-electrolytes 


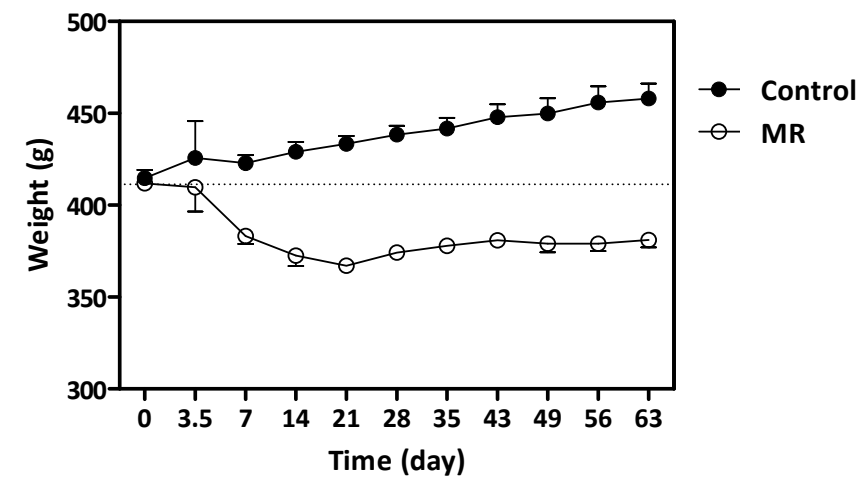

Fig. (5). Body weight of animals on control and MR diets. Data spans 9 weeks of the study and is reported as mean \pm SEM.

tested in MR rats including serum albumin, total protein content, serum creatinine and prealbumin (a sign of a significant deviation from normal food intake), remained at comparable levels to those in the control group. Arguably these changes in blood analyses and initial weight loss are not considered to be unhealthy.

The primary result of the study is that restricted dietary Met intake improved rat colonic epithelial barrier function with an onset as early as 2 weeks on MR diet and maintained at least for up to 9 weeks. We observed significant increases in the $R_{t}$ of distal colon tissue sheets from rats fed on MR diet compared to tissue from rats on control diet (Fig. 6A). This was accompanied by a significant reduction in the transepithelial flux of ${ }^{14} \mathrm{C}-D$-mannitol across distal colon in MR rats (Fig. 6B). Both parameters imply a decrease in colonic paracellular leak and an improvement in the TJ barrier function. Similar to what was observed in the LLC-PK 1 study, no significant changes in colon $\mathrm{I}_{\mathrm{scc}}$ were noted. This suggests a comparable $\mathrm{Na}^{+}$reabsorption or $\mathrm{Cl}^{-}$secretion from the colonocytes and that the difference seen in transepithelial $R_{t}$ was in fact the result of a difference in the TJ permeability, rather than altered transcellular permeability.

In addition to changes in TJ permeability, there are several other factors that may in part be responsible for the enhanced barrier function observed in rats on MR diet. The morphological architecture and cell proliferation of rat colon tissues were therefore evaluated by hematoxylin \& eosin staining and a semiquantitative Ki-67 staining, respectively. The gross histology, as well as the architecture of the crypts (i.e. length and frequency), and the general thickness of the colonic mucosa was similar in both control and MR groups. In both groups, the Ki-67 labeled cells were located in the appropriate compartments within the epithelium in a similar fashion, most densely right above the base of the crypts, indicating similar cell proliferation between the groups. There was no difference in the rate of apoptosis, or the observed mitotic frequency across the groups either. Overall, MR diet did not seem to alter the morphology or cell kinetics of the rat colonic mucosa.

We did see a moderate but significant decrease in the plasma and intracellular colonocyte Met levels in MR, which could possibly lead to a decreased degree of methylation of macromolecules including DNA. Since DNA methylation is one of the important epigenetic mechanisms known to alter
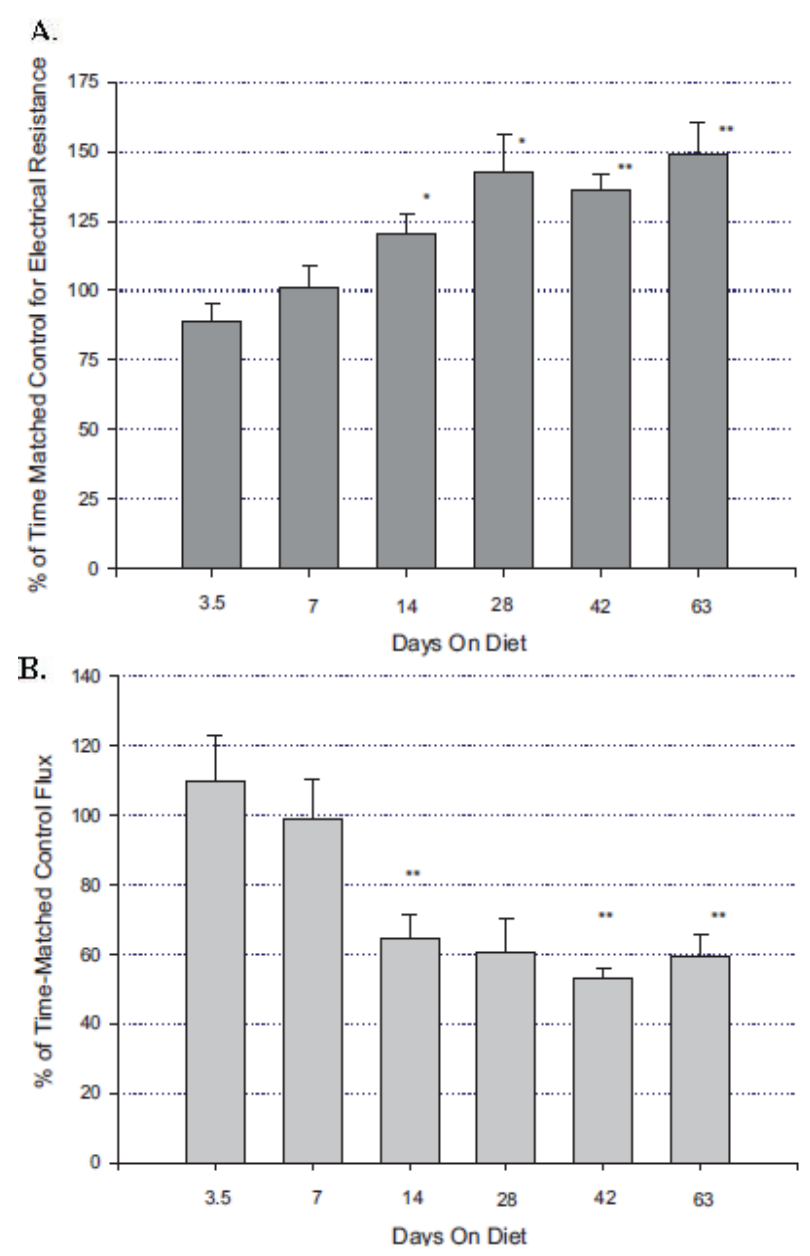

Fig. (6). Time course of MR-induced changes in rat colon epithelial barrier functions: (A) the relative change of $R_{t}$ compared to time-matched controls, and (B) transepithelial mannitol flux across the same tissues. Error bars indicate mean \pm SEM ( $\mathrm{n}=6$ to 12 separate time-matched tissues). $*$ and $* * *$ denote $\mathrm{p}<0.05$ and $\mathrm{p}<0.001$ significances, respectively, as tested by Student's $t$ test, assuming equal variances.

gene expression [38, 39], future work by our group will focus on the linkage between MR-related DNA methylation and $\mathrm{TJ}$ modifications.

Using RT-PCR technique, dietary MR was confirmed to affect the transcription of certain TJ genes. An $80 \%$ increase in claudin-3 mRNA was seen in rat colonic mucosal scrapes from rats on MR diet, while no significant change in occludin expression was noted (Fig. 7A). Western blot analysis also revealed an increase in claudin-3 protein abundance in MR compared to the control diet group (Fig. 7B). There was no significant change in claudin-1 or -7. Unlike LLC-PK 1 cells, claudin-2, $-4,-5$ and -6 were however not detectable in rat distal colon scrapes. Despite no change in occludin abundance, there is an additional occludin protein band, 3-5 $\mathrm{kDa}$ above the original occludin band, which suggests a possible post-translational modification of occludin protein by MR diet.

In summary, for the first time in an animal model, we demonstrated that restricted intake of Met improved colonic 
A.

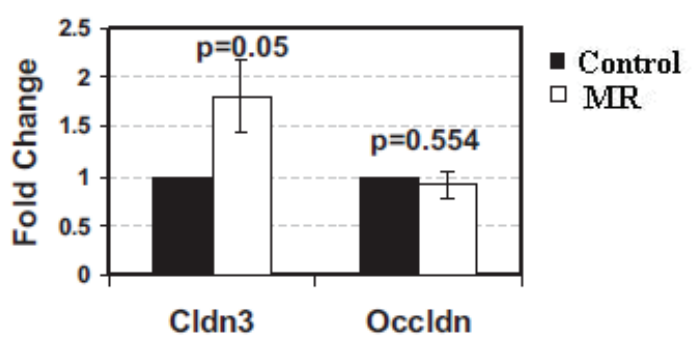

B.

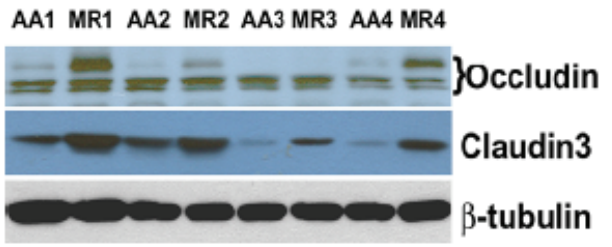

C.

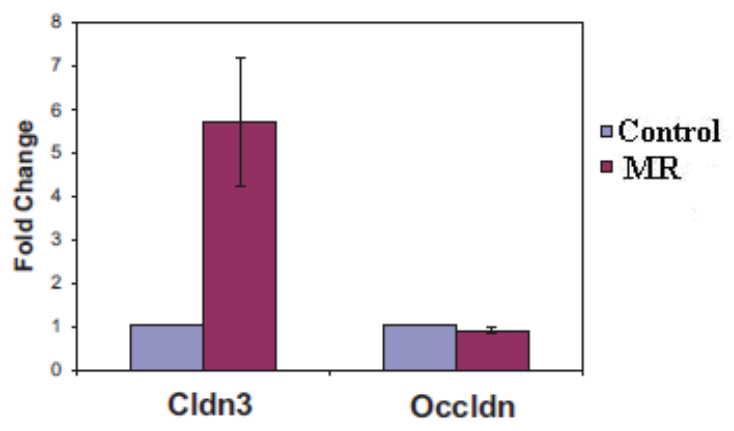

Fig. (7). Methionine restriction alters mRNA expression and protein abundance of certain $\mathrm{TJ}$ proteins in the distal colon of rats on control vs. MR diet. (A). RT-PCR of CLDN3 and OCLN expression as normalized with GAPDH value. Error bars indicate mean \pm SEM ( $n=3$ tissues); (B). Western blots analysis showing the steady-state level of $\mathrm{TJ}$ proteins (labels indicate the appropriate matched animal pairs) and (C). quantitation of claudin-3 and occludin levels as normalized with $\beta$-tubulin levels in each lane (mean \pm SEM, $\mathrm{n}=4$ tissues).

epithelial barrier function. It is worth pointing out that restricted Met intake may have different impact on other tissues and organs, depending on their specific TJs and TJ regulation pathways. For example, in parallel to the rat colon study, separate rat studies under the same control and MR conditions were carried out for 3 weeks to investigate the effect of MR on rat ileal tissue. In contrast to what was observed in rat colon, there was no significant change in $R_{t}, I_{s c c}$, or transepithelial flux values of tracers of two differences molecular weights $\left({ }^{14} \mathrm{C}-D\right.$-mannitol of $182 \mathrm{Da}$ and ${ }^{3} \mathrm{H}$ - $D$-lactulose of $342 \mathrm{Da}$ ) across distal ileal mucosal sheets. This suggests that dietary MR-induced barrier improvement might be tissue specific, thus each organ should be treated and studied differently.

\section{MR EFFECTS ON CLINICAL PATIENTS WITH CROHN'S DISEASE}

The intestinal barrier in patients with Crohn's disease is characterized by radiolabeled tracer leakage and lower electrical resistance and/or impedance, which in part can apparently be traced back to alterations in claudin proteins [40,
41]. In light of the ability of dietary MR to enhance epithelial barriers in both cell culture and rat models, a clinical study aiming to improve compromised GI barrier in patients with Crohn's disease was proposed and carried out. The 2-year study recruited 24 adult Crohn's disease patients, 17 of whom completed the study. Subjects in the trial were required to be on a 4-week meat- and fish-free diet with very sparing dairy, designed to lower dietary intake of Met by over $60 \%$. Low nitrogen intake as a result of the overall low protein consumption was corrected by supplementation with twice daily Hominex ${ }^{\circledR}-2$, a Met-free, amino acid-based nutrient formula produced by Abbott Labs. A small cohort of 5 patients was also randomized to take a sham diet with white meat and unrestricted dairy intake.

Crohn's disease activity indices (CDAIs) were computed for all patients at 3 different times: before the diet, at the end of the diet, and 4 weeks after resuming a normal diet. This score is a research tool utilized to quantify the symptoms of patients with Crohn's disease, and is considered to be the gold standard clinically for assessing Crohn's disease activity [42]. Results revealed a statistical decrease of the score (from $128 \pm 13$ to $106 \pm 11)(\mathrm{P}<0.02)$, showing a symptomatic improvement while on the diet. However, this could have been due to the elemental nature of a diet supplemented with Hominex ${ }^{\circledR}-2$, rather than a decreased Met level. It should also be noted that the near vegan nature of the diet involved a voluntary increase in plant protein intake, and hence roughage, which may not have been salutary, given these patients' condition with a chronic inflamed gut. Patients on the sham diet (without red meat, but allowing chicken, fish and dairy) showed no significant change in CDAI scores before vs. end of the diet.

In contrast to what was seen in cell culture and rat study, amino acid analysis of plasma Met revealed a merely $10 \%$ non-significant reduction at the end of the 4-week diet as compared to their pre-diet level. The lack of an effect of the MR diet on plasma Met concentration suggests that either the dietary MR was not stringent enough, and /or the cellular concentrative Met transport systems can overcome this level of decreased intake, and/or the human body can hydrolyze labile protein stores, possibly in the liver and kidney, to make up Met intake deficit. With this being said, we did observe a small (12\%) but significant decrease in plasma Met seen in a previous trial with 5 healthy control subjects on the same dietary MR plan. In neither group did we observe a significant change in body weight while on the diet. In comparison, an approximately $40 \%$ decrease of plasma Met was previously reported on the first day of a Met-free regimen, which was a more stringent, formulabased Met-free diet [12].

A significant (17\%) decrease in serum homocysteine was however observed among Crohn's patients in our trial. A recent report studying sulfur amino acids in MR rats using a similar diet to the rat chow that was used in our studies revealed a near $150 \%$ increase in serum homocysteine level [43]. It was suspected that there occurred a possible MRinduced inhibition of homocysteine trans-sulfuration due to the MR diet. This discrepancy could be due to a species difference, and/or the fact that laboratory rats are more compliant to the MR diet, and/or an improved vitamin B 
intake with the Hominex ${ }^{\circledR}-2$ supplement to the Crohn's patients who may normally have impaired ileal vitamin B absorption.

The unchanged plasma Met concentration was accompanied by an insignificant, yet $20 \%$ increase of urinary lactulose/mannitol (L/M) ratio, which was due to an increased lactulose leak in over $60 \%$ of patients with mannitol leak staying constant. This may relate to Crohn's patients, having a chronically inflamed GI tract in need of supra-normal rates of tissue regeneration and repair. Despite an unaltered plasma Met level, it is still possible that the intracellular enterocyte Met level was affected. If this was the case the MR diet could pose a contraindication for their condition by decreasing rates of protein synthesis and cell restitution.

Most of our patients enrolled in the study had only mild-moderate disease, with roughly half receiving TNF immunomodulatory medications. Overall, Crohn's patients and healthy controls, on either MR or sham diet, tolerated the regimen and managed their disease well throughout the trial period.

\section{SUMMARY}

With evidence more than ever revealing the actions of disease pathogens and mediators on TJs, sculpturing a more resilient barrier by minimizing junctional leakiness may prove to be an exceedingly beneficial way to prevent and/or treat certain diseases. Dietary modifications, such as MR appear to be one of the modalities that show promise in offsetting disease-introduced barrier leakiness. In our studies using cell culture and animal models, we demonstrated that dietary MR can indeed remodel TJs and improve barrier function. Implementing these findings clinically will clearly require many further studies focusing on dietary implementation, target-tissues, and targeted disease states. In addition, a thorough insight into the molecular mechanism of MR is also needed. Such understanding may then lead to more practical dietary approaches to barrier enhancement than MR itself.

\section{CONFLICT OF INTEREST}

None declared.

\section{ABBREVIATIONS}

$\begin{array}{ll}\mathrm{CR} & =\text { Caloric restriction } \\ \mathrm{GI} & =\text { Gastrointestinal } \\ \mathrm{I}_{\text {scc }} & =\text { Short-circuit current } \\ \mathrm{Met} & =\text { Methionine } \\ \mathrm{MR} & =\text { Methionine restriction } \\ \mathrm{R}_{\mathrm{t}} & =\text { Transepithelial electrical resistance } \\ \mathrm{SAM} & =\text {-adenosylmethionine } \\ \mathrm{TJ} & =\text { Tight junction }\end{array}$

\section{ACKNOWLEDGEMENT}

This work was supported in part by grants from the Sharpe/Strumia Foundation and the Broad Foundation.

\section{REFERENCE}

[1] McCay CM, Cromwell MF, Maynard LA. The effect of retarded growth upon length of life span and upon the ultimate body size. $\mathrm{J}$ Nutr 1935; 10: 63-79.

[2] Masoro EJ. Caloric restriction-induced life extension of rats and mice: a critique of proposed mechanisms. Biochim Biophys Acta 2009; 1790: 1040-8.

[3] Grandison RC, Piper MD, Partridge L. Amino-acid imbalance explains extension of lifespan by dietary restriction in Drosophila. Nature 2009; 462: 1061-4.

[4] Mullin JM, Skrovanek SM, Valenzano MC. Modification of tight junction structure and permeability by nutritional means. Ann NY Acad Sci 2009; 1165: 99-112.

[5] Hoffman RM. Altered methionine metabolism and transmethylation in cancer. Anticancer Res $1985 ; 5: 1-30$.

[6] Fu YM, Zhang H, Ding M, et al. Specific amino acid restriction inhibits attachment and spreading of human melanoma via modulation of the integrin/focal adhesion kinase pathway and actin cytoskeleton remodeling. Clin Exp Metastasis 2004; 21: 587-98.

[7] Lu S, Chen GL, Ren C, Kwabi-Addo B, Epner DE. Methionine restriction selectively targets thymidylate synthase in prostate cancer cells. Biochem Pharmacol 2003; 66: 791-800.

[8] Tisdale M, Eridani S. Methionine requirement of normal and leukaemic haemopoietic cells in short term cultures. Leuk Res 1981; 5: 385-94.

[9] Komninou D, Leutzinger Y, Reddy BS, Richie JP Jr. Methionine restriction inhibits colon carcinogenesis. Nutr Cancer 2006; 54: 202-8.

[10] Epner DE. Can dietary methionine restriction increase the effectiveness of chemotherapy in treatment of advanced cancer? J Am Coll Nutr 2001; 20: 443S-9S; discussion 73S-75S.

[11] Epner DE, Morrow S, Wilcox M, Houghton JL. Nutrient intake and nutritional indexes in adults with metastatic cancer on a phase I clinical trial of dietary methionine restriction. Nutr Cancer 2002; 42: $158-66$.

[12] Durando X, Thivat E, Farges MC, et al. Optimal methionine-free diet duration for nitrourea treatment: a Phase I clinical trial. Nutr Cancer 2008; 60: 23-30.

[13] Durando X, Farges MC, Buc E, et al. Dietary Methionine Restriction with FOLFOX Regimen as First Line Therapy of Metastatic Colorectal Cancer: A Feasibility Study. Oncology 2010; 78: 205-9.

[14] Fu YM, Yu ZX, Lin H, Fu X, Meadows GG. Selective amino acid restriction differentially affects the motility and directionality of DU145 and PC3 prostate cancer cells. J Cell Physiol 2008; 217: 184-93.

[15] Fu YM, Yu ZX, Ferrans VJ, Meadows GG. Tyrosine and phenylalanine restriction induces $\mathrm{G} 0 / \mathrm{G} 1$ cell cycle arrest in murine melanoma in vitro and in vivo. Nutr Cancer 1997; 29: 104-13.

[16] Fu YM, Yu ZX, Li YQ, et al. Specific amino acid dependency regulates invasiveness and viability of androgen-independent prostate cancer cells. Nutr Cancer 2003; 45: 60-73.

[17] Fu YM, Zhang H, Ding M, et al. Selective amino acid restriction targets mitochondria to induce apoptosis of androgen-independent prostate cancer cells. J Cell Physiol 2006; 209: 522-34.

[18] Fu YM, Lin H, Liu X, Fang W, Meadows GG. Cell death of prostate cancer cells by specific amino acid restriction depends on alterations of glucose metabolism. J Cell Physiol 2010 Apr 2.

[19] Stanfel MN, Shamieh LS, Kaeberlein M, Kennedy BK. The TOR pathway comes of age. Biochim Biophys Acta 2009; 1790: 1067-74.

[20] Hwang JT, Kwon DY, Yoon SH. AMP-activated protein kinase: a potential target for the diseases prevention by natural occurring polyphenols. N Biotechnol 2009; 26: 17-22.

[21] Pallas M, Verdaguer E, Tajes M, Gutierrez-Cuesta J, Camins A. Modulation of sirtuins: new targets for antiageing. Recent Pat CNS Drug Discov 2008; 3: 61-9.

[22] Dilova I, Easlon E, Lin SJ. Calorie restriction and the nutrient sensing signaling pathways. Cell Mol Life Sci 2007; 64: 752-67.

[23] Houtkooper RH, Williams RW, Auwerx J. Metabolic networks of longevity. Cell 2010; 142: 9-14.

[24] Loenen WA. S-adenosylmethionine: jack of all trades and master of everything? Biochem Soc Trans 2006; 34: 330-3.

[25] Schneeberger EE, Lynch RD. The tight junction: a multifunctional complex. Am J Physiol Cell Physiol 2004; 286: C1213-28.

[26] Ikenouchi J, Furuse M, Furuse K, Sasaki H, Tsukita S. Tricellulin constitutes a novel barrier at tricellular contacts of epithelial cells. J Cell Biol 2005; 171: 939-45. 
[27] Skrovanek S, Valenzano MC, Mullin JM. Restriction of sulfurcontaining amino acids alters claudin composition and improves tight junction barrier function. Am J Physiol Regul Integr Comp Physiol 2007; 293: R1046-55.

[28] Mullin JM, Weibel J, Diamond L, Kleinzeller A. Sugar transport in the LLC-PK1 renal epithelial cell line: similarity to mammalian kidney and the influence of cell density. J Cell Physiol 1980; 104: 375-89.

[29] Oliveira SS, Morgado-Diaz JA. Claudins: multifunctional players in epithelial tight junctions and their role in cancer. Cell Mol Life Sci 2007; 64: 17-28.

[30] Mullin JM, Agostino N, Rendon-Huerta E, Thornton JJ. Keynote review: epithelial and endothelial barriers in human disease. Drug Discov Today 2005; 10: 395-408.

[31] Amasheh M, Andres S, Amasheh S, Fromm M, Schulzke JD. Barrier effects of nutritional factors. Ann NY Acad Sci 2009; 1165 : 267-73.

[32] Richie JP Jr, Komninou D, Leutzinger Y, et al. Tissue glutathione and cysteine levels in methionine-restricted rats. Nutrition 2004; 20: 800-5.

[33] Richie JP Jr, Leutzinger Y, Parthasarathy S, Malloy V, Orentreich $\mathrm{N}$, Zimmerman JA. Methionine restriction increases blood glutathione and longevity in F344 rats. FASEB J 1994; 8: 1302-7.

[34] Ramalingam A, Wang X, Gabello M, et al. Dietary Methionine Restriction Improves Colon Tight Junction Barrier Function and Alters Claudin Expression Pattern. Am J Physiol Cell Physiol 2010 Aug 25.

[35] Malloy VL, Krajcik RA, Bailey SJ, Hristopoulos G, Plummer JD, Orentreich N. Methionine restriction decreases visceral fat mass and preserves insulin action in aging male Fischer 344 rats independent of energy restriction. Aging Cell 2006; 5: 305-14.

[36] Orentreich N, Matias JR, DeFelice A, Zimmerman JA. Low methionine ingestion by rats extends life span. J Nutr 1993; 123: 269-74.

[37] Hasek BE, Stewart LK, Henagan TM, et al. Dietary methionine restriction enhances metabolic flexibility and increases uncoupled respiration in both fed and fasted states. Am J Physiol Regul Integr Comp Physiol 2010 Jun 10.

[38] Tost J. DNA methylation: an introduction to the biology and the disease-associated changes of a promising biomarker. Methods Mol Biol 2009; 507: 3-20.

[39] Miranda TB, Jones PA. DNA methylation: the nuts and bolts of repression. J Cell Physiol 2007;213: 384-90.

[40] Zeissig S, Burgel N, Gunzel D, et al. Changes in expression and distribution of claudin 2, 5 and 8 lead to discontinuous tight junctions and barrier dysfunction in active Crohn's disease. Gut 2007; 56: 61-72.

[41] Zeissig S, Bojarski C, Buergel N, et al. Downregulation of epithelial apoptosis and barrier repair in active Crohn's disease by tumour necrosis factor alpha antibody treatment. Gut 2004; 53: 1295-302.

[42] Jorgensen LG, Fredholm L, Hyltoft Petersen P, Hey H, Munkholm $\mathrm{P}$, Brandslund I. How accurate are clinical activity indices for scoring of disease activity in inflammatory bowel disease (IBD)? Clin Chem Lab Med 2005; 43: 403-11.

[43] Elshorbagy AK, Valdivia-Garcia M, Refsum H, Smith AD, Mattocks DA, Perrone CE. Sulfur amino acids in methionine-restricted rats: Hyperhomocysteinemia. Nutrition 2010 Jan 15.

Received: June 30, 2011

Revised: August 08, 2011

Accepted: August 22, 2011

(C) Wang and Mullin; Licensee Bentham Open.

This is an open access article licensed under the terms of the Creative Commons Attribution Non-Commercial License (http://creativecommons.org/licenses/ by-nc/3.0/) which permits unrestricted, non-commercial use, distribution and reproduction in any medium, provided the work is properly cited. 